\title{
Research on Short-Term Wind Power Prediction Based on Combined Forecasting Models
}

\author{
Chi Zhang ${ }^{1}$, Jie Zeng ${ }^{1}$, Ning Xie ${ }^{1}$, Ping Yang ${ }^{2,3}$, Yujia Zhang ${ }^{2,3}$ and Zhen Zhang ${ }^{4}$ \\ ${ }^{1}$ Electric Power Research Institute, Guangdong Power Grid Corporation, 510640, Guangzhou, China \\ ${ }^{2}$ School of Electric Power, South China University of Technology, 510640, Guangzhou, China \\ ${ }^{3}$ Guangdong Key Laboratory of Clean Energy Technology, South China University of Technology, 511458, Guangzhou, China \\ ${ }^{4}$ Guangdong Intework Energy Technology Co., Ltd, 511458, Guangzhou, China
}

\begin{abstract}
Short-Term wind power forecasting is crucial for power grid since the generated energy of wind farm fluctuates frequently. In this paper, a physical forecasting model based on NWP and a statistical forecasting model with optimized initial value in the method of BP neural network are presented. In order to make full use of the advantages of the models presented and overcome the limitation of the disadvantage, the equal weight model and the minimum variance model are established for wind power prediction. Simulation results show that the combination forecasting model is more precise than single forecasting model and the minimum variance combination model can dynamically adjust weight of each single method, restraining the forecasting error further.
\end{abstract}

\section{Introduction}

Over the past 10 years, the wind power has been developing rapidly. By the end of 2013, the world's wind-power capacity exceeded 290GW [1], [2]. However, the generated energy of wind farm is fluctuating, which brings great difficulties to grid-connection. In order to guarantee the reliability of power grid, an accurate method for wind power prediction must be found.

At present, there are two major methods for the windpower prediction: physical forecasting model and statistical forecasting model. Physical forecasting model established mainly based on NWP (Numerical Weather Prediction) [3], transferring the wind speed and the wind direction of NWP into those of Turbine hub height, helps forecasting the wind power of each wind turbine [4], [5]. The main methods of statistical prediction commonly contains several methods, such as time series method, improved time series method [6], Feedforward Neural Network (FNN) [7], Regression Neural Network, Back propagation Neural Network (BP) [8], Support Vector Machine (SVM) [9], grey theory, genetic algorithm and so on.

Both physical and statistical methods have their own advantages and disadvantages. Combination model put different prediction model together through the appropriate combination method, in order to utilize a single model's merit and overcome the limitations of the disadvantage, so as to improve the accuracy of prediction. The most critical problem in combination forecasting model is how to set the weight of each single model for making the accuracy of prediction higher [10]. In this paper, we present a wind power combination forecasting method, combining physical model and statistical model. This combination forecasting model uses minimum variance method to determine the weight of each single model to obtain the wind power forecasting value [11]. This method combines the advantages of physical model and statistical model, which leads to higher wind power forecasting accuracy.

\section{Short-Term wind power forecasting method}

\subsection{Physical wind power forecasting model}

The physical wind power forecasting model tries to obtain the wind speed of each turbine hub from NWP data. Based on physical model, the wind power prediction method includes wind-speed transformation model, wake model and wind speed-power curve.

In this paper, the format of the NWP is WRF, whose spatial resolution is 27 kilometers and the temporal resolution is 15 minutes. WRF, researched and developed by the USA, is the world's advanced meteorological numerical model at present.

The wind-speed transformation model uses Inverse Distance Weighted function to transform wind speed of NWP into that of wind turbine. The Inverse Distance Weighted function is shown as follows.

$$
v^{*}=\sum \frac{1 / l_{i}}{\sum 1 / l_{i}} v_{i}
$$


where $l_{i}$ is the distance between the position of NWP data and that of wind turbine, $v^{*}$ is wind speed of the $i^{\text {th }}$ wind turbine, $v_{i}$ is wind speed of correlative NWP data.

Considering the wind shear, the power function is used to calculate wind speed at the height of hub. The power function in this paper is shown as follows.

$$
v_{2}=v_{1} \frac{\ln h_{2}-\ln h_{0}}{\ln h_{1}-\ln h_{0}}
$$

In the actual wind farm, the position of each turbine is different. It is obviously that the downwind wind turbine will receive less wind resource than upwind wind turbine. This phenomenon is called wake effects.

When the wind is absorbed by the wind turbine, the wind speed of the wind turbine will be lower than the wind speed of the wind turbine. By improving the Jensen model, we can get the wind speed of the actual wind turbine hub height. According to improved Jensen model, we calculate the actual wind speed reaching the hub of each wind turbine.

At last, the wind power of each wind turbine can be calculated through wind speed-power curve.

The complete flowchart of physical wind power forecasting model based on NWP is shown as follows.

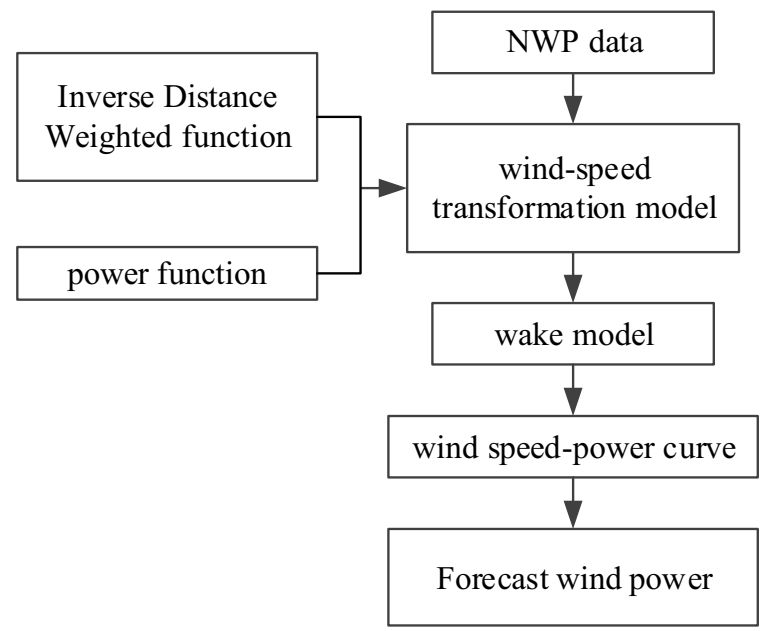

Figure 1. Flowchart of physical wind power forecasting model.

\subsection{Statistical wind power forecasting model}

In this paper, the MIV-BP method is used to forecast wind power.

First, BP neural network is used to calculate the Mean Impact Value (MIV) of each input variable. Then, the variables will be sorted in order to find the key input items. At last, the BP the neural network is trained with the selected key items as the input variables and the actual power as the output variable.

The process of calculating Impact Value Mean (MIV) is as follows.

First, we use the training set to get the training model, increasing and decreasing each training input variable values by $10 \%$ to constitute two new sample set. Taking the two new sample sets as simulation samples, the trained model is utilized to calculate the output value. The Subtraction of two output results of the two data sets is the output value of the Impact Value (IV), the mean value of the IV of each variable is Mean Impact Value (MIV). Finally, in order to find the relative influence of each variable, the MIV value of each variable is seriously ordered. According to the relative influence, we can pick the input variables of neural network out.

The complete flowchart based on MIV-BP is shown as follows.

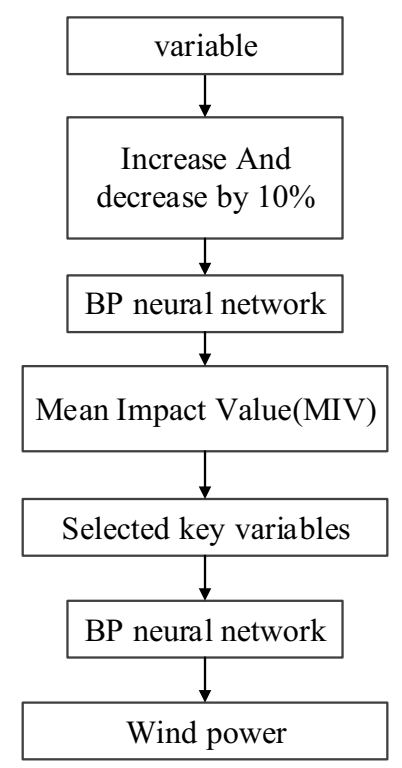

Figure 2. Flowchart of MIV-BP model.

\section{Combination wind power forecasting method}

\subsection{Equal weight combination model}

The most common and simple combination forecasting algorithm is equal weight method. Assuming there exist a single forecasting model whose number is $\mathrm{m}$. The wind power forecasted of the $i$ model at the time of $J$ is $\hat{y}_{j i}$ and the actual wind power at the time of $J$ is $y_{j}$. Then the forecasting value of equal weight method at the time of $J$ is shown as follows.

$$
\hat{y}_{j}=\frac{1}{m} \sum_{i=1}^{m} \hat{y}_{j i}
$$

The relative error of the equal-weight combined forecasting method is shown as follows.

$$
\delta_{\text {mean }}=\frac{\hat{y}_{j}-y_{j}}{y_{j}}
$$

According to the upper formula, equal-weight combined forecasting method is trying to reduce the prediction error of the final power by averaging the forecasting value of single forecasting methods. Equal weight which is simple, combined with forecasting method can reduce the random error of the forecasting model to some extent. The problem of equal weight model is that the weight of each single model cannot be adjusted dynamically according to the difference between the predicted value and the actual value. So the improved space of this algorithm is not good as other algorithms. 
Due to the characteristics of the equal weight combination forecasting model, the weight of combination model is used as a benchmark to evaluation the performance of the other algorithms.

\subsection{Minimum variance combination model}

The minimum variance combination forecasting model can solve the problem of equal weight method which cannot be adjusted dynamically. The minimum variance method can get higher accuracy than equal weight method.

Assuming that there are $m$ different forecasting models, the forecasting wind power of the $i$ model at the time of $J$ is $\hat{y}_{j i}$ and the actual wind power at the time of $J$ is $y_{j}$. The weight of the $i$ model is $w_{i}$. Then the following constraints need to be satisfied.

$$
\left\{\begin{array}{c}
\sum_{i=1}^{m} w_{i}=1 \\
w_{i} \geq 0, i=1, \ldots, m
\end{array}\right.
$$

The forecasting value at the time of $J$ is shown as follows.

$$
\hat{y}_{j}=\sum_{i=1}^{m} w_{i} * \hat{y}_{j i}
$$

The error of forecast at the time of $J$ is shown as follows.

$$
\delta_{j}=\hat{y}_{j}-y_{j}
$$

In order to determine the weight coefficient $w_{i}$, the forecasting value and actual value are used to fitting the weight coefficient $w_{i}$. This paper uses the minimum variance as the objective function. The expression of objective function and constraints is shown as follows.

$$
\left\{\begin{array}{c}
\min Z=\sum_{j=1}^{n} \delta_{j}^{2} \\
s t: \sum_{i=1}^{m} w_{i}=1 \\
w_{i} \geq 0, i=1, \ldots, m
\end{array}\right.
$$

where $n$ is the number of actual value and $m$ represent the number of single forecasting model.

The formula shown above is a quadratic programming problem, which can be written as the following form.

$$
\left\{\begin{array}{c}
\min f(x)=\frac{1}{2} x^{T} G x+r^{T} x\left(x \in R^{n}\right) \\
s t: A_{i}^{T} x-b_{i}=0,(i=1, \ldots, m) \\
A_{i}^{T} x-b_{i} \geq 0,(i=m+1, \ldots, m+l)
\end{array}\right.
$$

where $G$ is n-orders symmetric matrix, $r, A_{i}(i=1,2, \ldots$, $m+l)$ is n-dimensional column vector and $b_{i}(i=1,2, \ldots$, $m+l)$ is real number.

The objective function can be expanded as follows.

$$
\begin{aligned}
\min Z & =\sum_{j=1}^{n}\left(\hat{y}_{j}-y_{j}\right)^{2}=\sum_{j=1}^{n}\left(\sum_{i=1}^{m} w_{i} *\left(\hat{y}_{i j}-y_{i j}\right)\right)^{2}=\sum_{j=1}^{n}\left(\sum_{i=1}^{m} w_{i} * \delta_{i j}\right)^{2} \\
& =\sum_{i=1}^{m}\left[w_{i}^{2}\left(\sum_{j=1}^{n} \delta_{i j}^{2}\right)\right]+2 \sum_{1 \leq k, j \leq m, k \neq j}\left[w_{i} * w_{k} * \sum_{j=1}^{n}\left(\delta_{i j} * \delta_{k j}\right)\right]
\end{aligned}
$$

Simplifying the formula above, we need to set:

$$
\left\{\begin{array}{c}
g_{j k}=g_{k j}=\sum_{j=1}^{n}\left(\delta_{i j} * \delta_{k i}\right),(j, k=1,2, \ldots, m) \\
G=\left[\begin{array}{ccc}
g_{11} & \ldots & g_{1 m} \\
\ldots & \ldots & \ldots \\
g_{m 1} & \ldots & g_{m m}
\end{array}\right] \\
W=\left(w_{1}, w_{2}, \ldots, w_{m}\right)^{T} \\
e=(1,1, \ldots, 1)^{T}
\end{array}\right.
$$

Then, the expression of objective function and constraints is shown as follows.

$$
\left\{\begin{array}{c}
\min Z=W^{T} G W \\
e^{T} W-1=0 \\
W^{T} \geq 0
\end{array}\right.
$$

The extreme value of the above formula can be calculated through the Lagrange function. The $N$ actual power and $N$ forecasting power is constantly updated as time goes by. Moreover, $w_{i}$ can also be constantly updated. The wind power sequence is a time series with very strong temporal correlation. Thus, by constantly updating the last $N$ data and determine the weight $w_{i}$ at different times, we will effectively improve the forecasting accuracy.

\section{Simulation verifications}

\subsection{Initial data}

In this case, the format of NWP data is WRF. The actual output data of wind farm is provided by the Power Grid Corp. The selected data is active power of high voltage side in the No. 1 main transformer, whose time interval is 15 minutes. The observed data vary from 00:00:00 201301-01 to 23:45:00 2013-07-31. The latitude and longitude of NWP points is shown as follows.

Table 1. Latitude and longitude of NWP points.

\begin{tabular}{cccc}
\hline longitude & 108.42 & 108.39 & 108.37 \\
\hline Latitude & 18.79 & 19.02 & 19.25 \\
\hline
\end{tabular}

The spatial interval of WRF data is 27 kilometers, and the temporal resolution of WRF data is 15 minutes. In this wind farm, there are 24 wind turbines, whose rated capacity are all $1500 \mathrm{~kW}$ and rated wind speed $12 \mathrm{~m} / \mathrm{s}$ and the height of wind turbines are 70 meters.

Combination forecasting model combining physical forecasting model and BPNN model is used to forecast wind power and compare the accuracy with that of single physical forecasting model and BPNN model in this case. 


\subsection{Result analysis of single forecast method}

In this case, we analyzed the forecast result of two single models shown above. The results of physical forecasting methods based on WRF and statistical forecasting methods with BP neural network are shown as follows.

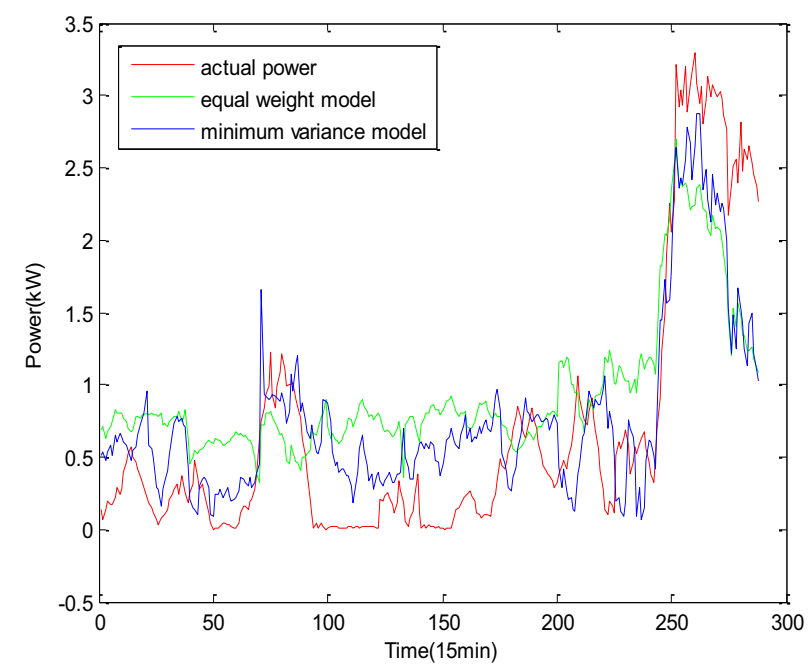

Figure 3. Forecasting results of single model.

The forecasting error curves of physical forecasting methods based on WRF and statistical forecasting methods with BP neural network are shown as follows.

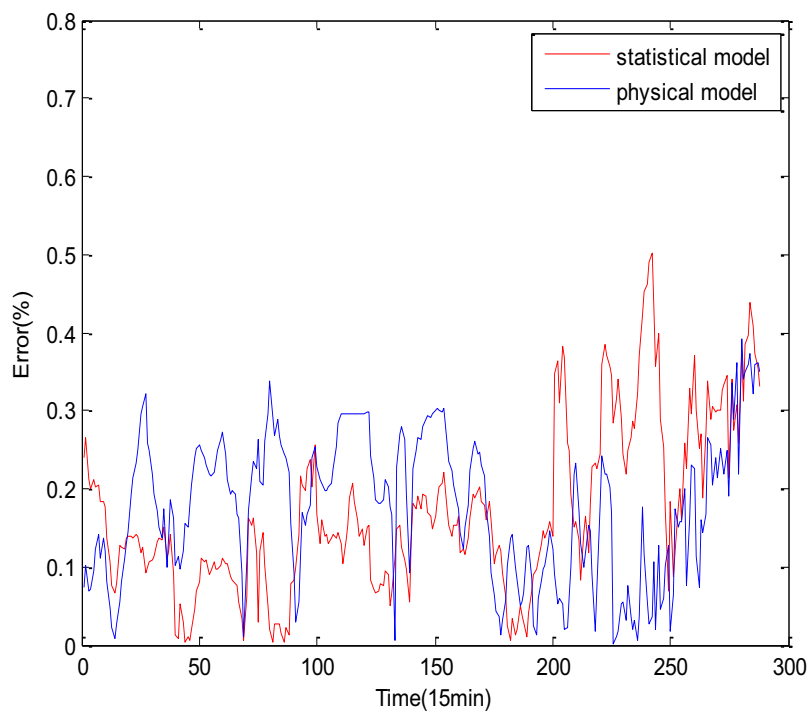

Figure 4. Forecasting error curves of single model.

\subsection{Result analysis of combination forecast method}

In this case, the combined forecasting method is used to predict wind power by combining the physical forecasting methods based on WRF and statistical forecasting methods with BP neural network.

In equal weight combination model, the weights of two methods are both 0.5. In minimum variance combination model, the weights of two methods are changing dynamically.

The forecasting result of combination method is shown as follows.

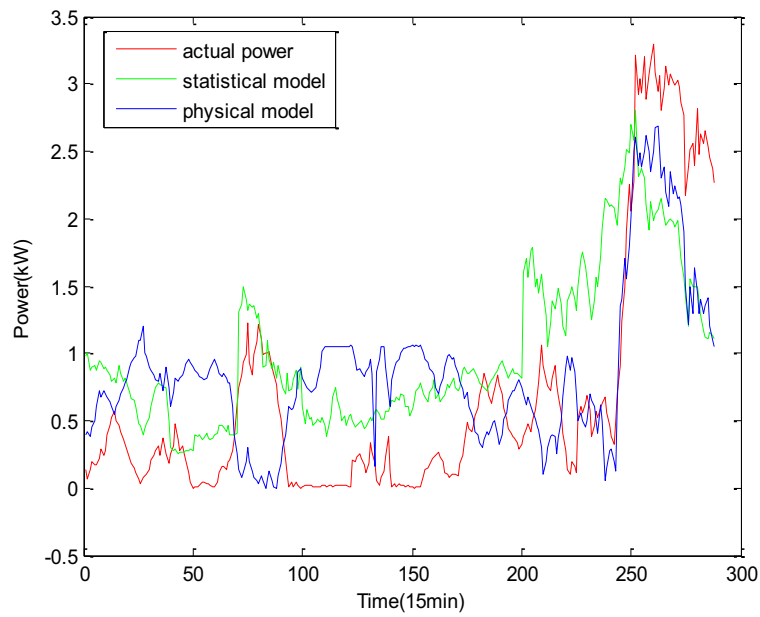

Figure 5. Forecasting results of combination model.

The forecasting error curves of equal weight combination method and minimum variance combination method are shown as follows.

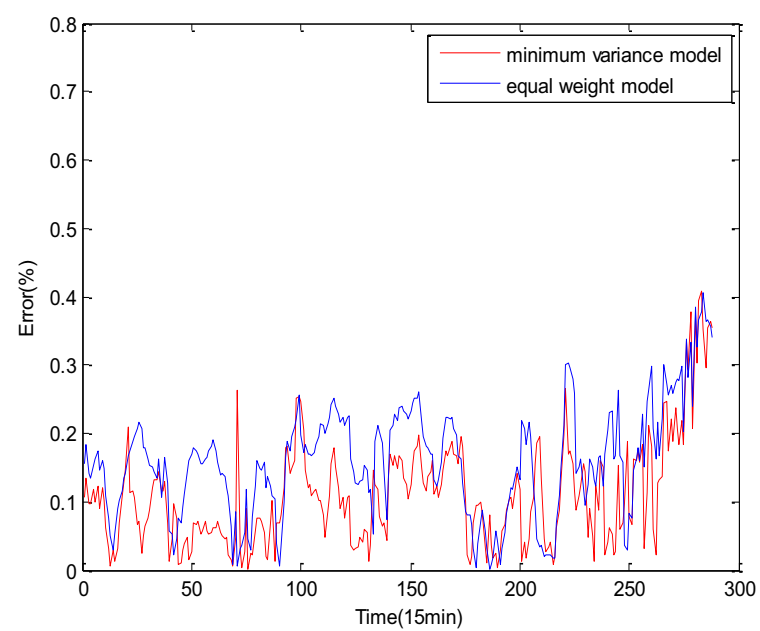

Figure 6. Forecasting error curves of combination model.

The forecasting Root-Mean-Square Error (RMSE) of combination method, physical method and statistical method is shown as follows.

Table 2. RMSE of forecasting method.

\begin{tabular}{cc}
\hline Forecasting method & RMSE \\
\hline equal weight combination method & $16.21 \%$ \\
minimum variance combination method & $15.26 \%$ \\
physical method based on WRF & $19.62 \%$ \\
BP neural network & $18.6 \%$ \\
\hline
\end{tabular}

It can be seen from the above that equal weight combination method cannot adjust its weight according to the error of the previous forecast namely it cannot improve forecast accuracy significantly. However, the equal weight combination method also behaves better than single forecasting method though it is the most simple combination method, which shows that the combination forecasting method is a direction of improving accuracy.

It concludes that the minimum variance combination forecasting method can make full use of the wind farm output temporal correlation information and adjust the weight of each simple method, which reduces forecasting 
error greatly, simultaneously, the minimum variance method also has its own limitation. According to the picture above, we consider that the minimum variance results can't follow the actual power when the actual power varies widely for the adjustment of minimum variance method lags behind the changes of actual power, which causes that the minimum variance method can't effectively reduce the forecasting error in the case of widely changes of output power.

\section{Conclusions}

Due to the nonlinear and unstable character of wind power, wind power forecasting is a tough and complicated work in the application of wind. In this paper, according to various wind power prediction mathematical model, we build two kinds of single prediction model based on NWP physical forecasting model and the model with optimized initial value in the method of BP neural network. For integrating the advantages of these models, we formulate the combination forecasting algorithm of equal weight model and minimum variance model. Simulations show as follows:

1) The physical forecasting model based on NWP keeps away from the limitation of a large amount of historical data while the prediction accuracy is relatively low. The statistical model based on BP neural network needs to accumulate a large amount of historical data to maintain high prediction accuracy.

2) The combined forecasting model can improve the forecasting accuracy for reducing the accidental error caused by single forecasting model.

3) The minimum variance combination forecasting model can adjust the weight of each simple model according to the accumulated error, which can achieve higher accuracy combined with equal weight combination forecasting model.

\section{Acknowledgments}

This work was supported by Guangdong Strategic Emerging Industry Core Technology Research Projects (2012A032300013); National Science and Technology Support Program (2015BAA06B02).

\section{References}

1. M. A. Islam, et al., Global renewable energy-based electricity generation and smart grid system for energy security. Scientific World Journal, 2014: 197136, (2014)

2. World Wind Energy Association, Half-Year Report, (2013)

3. E. Pelikán, et al., Wind power forecasting by an empirical model using NWP outputs, in Environment and Electrical Engineering (EEEIC), 2010 9th International Conference on, 2010, IEEE: Prague, Czech Republic, p. 45

4. S. Al-Yahyai, A. Gastli, Y. Charabi, Probabilistic wind speed forecast for wind power prediction using pseudo ensemble approach, in Power and Energy (PECon), 2012 IEEE International Conference on. 2012, IEEE: Kota Kinabalu, p. 127

5. S. Buhan, I. Cadirci, Multi-Stage Wind-Electric Power Forecast by Using a Combination of Advanced Statistical Methods. Industrial Informatics, IEEE Transactions on, (99):1, (2015)

6. J. Taylor, P. McSharry, Short-Term load forecasting methods: An evaluation based on European data, IEEE Trans. Power Syst., 22(4):2213-2219, (Nov. 2007)

7. H. Quan, D. Srinivasan, A. Khosravi, Short-Term load and wind power forecasting using neural network-based prediction intervals, IEEE Trans Neural Netw Learn Syst,. 25(2):303-15, (2014)

8. H. Quan, D. Srinivasan, A. Khosravi, Incorporating Wind Power Forecast Uncertainties Into Stochastic Unit Commitment Using Neural Network-Based Prediction Intervals, IEEE Trans. Neural. Netw. Learn. Syst., (2014)

9. J. Wen, et al., Short-Term wind power forecasting based on lifting wavelet transform and SVM, in Power Engineering and Automation Conference (PEAM), 2012 IEEE, IEEE: Wuhan, p. 1, (2012)

10. M. B. Ozkan, P. Karagoz, A Novel Wind Power Forecast Model: Statistical Hybrid Wind Power Forecast Technique (SHWIP), Industrial Informatics, IEEE Transactions on, 11(2):375, (2015)

11. L. Landberg, Short-Term prediction of local wind conditions, No.Ris Ø-R-702(EN), RisØ National Laboratory, RisØ, Denmark, (1994) 